\title{
Biped Robot Research at Waseda University
}

\author{
Kenji Hashimoto \\ Research Institute for Science and Engineering, Waseda University, \\ \#41-304, 17 Kikui-cho, Shinjuku-ku, Tokyo 162-0044, Japan \\ Atsuo Takanishi \\ Department of Modern Mechanical Engineering, Waseda University, \\ TWIns Room\# 3C-202, 2-2, Wakamatsu-cho, Shinjuku-ku, Tokyo 162-8480, Japan \\ E-mail: contact@takanishi.mech.waseda.ac.jp \\ www.takanishi.mech.waseda.ac.jp/top/index.htm
}

\begin{abstract}
Waseda University has researched on biped robots since 1967. This paper describes our latest biped robots: (i) WABIAN-2, (ii) a biped running robot, and (iii) WL-16. WABIAN-2 is a biped humanoid robot and has realized a human-like walk with the knees stretched by utilizing a 2-DOF waist mimicking a human's pelvic motion. We are developing a new biped humanoid robot which can jump and run by utilizing a pelvic movement and leg elasticity. WL-16 is a human-carrying biped vehicle.
\end{abstract}

Keywords: Biped walking, Running, Humanoid robot, Legged robot, Walking chair

\section{Introduction}

Waseda University has researched on biped robots since 1967 and has been one of the leading research sites for humanoid robots since the late Prof. Ichiro Kato and his colleagues started the WABOT Project in 1970. We have developed a variety of humanoid robots including WABOT-1 which is the first full-scale human-like robot made in 1973 and the biped humanoid robot WABIAN in 1997.

This paper describes our latest biped robots, WABIAN-2 ${ }^{1}$ (WAseda BIpedal humANoid - No. 2), a running robot $^{2}$ and WL-16 ${ }^{3}$ (Waseda Leg - No. 16).

\section{Biped Humanoid Robot, WABIAN-2}

We have developed a biped humanoid robot named WABIAN-2 as a human motion simulator to mimic human's motions and mechanisms (see Fig. 1).
WABIAN-2 has 41 degrees of freedom (DoF) (a 3DoF neck, a 2-DoF trunk, two 7-DoF arms, two 3-DoF hands, a 2-DoF waist, two 6-DoF legs and two 1-DoF feet having passive toe joint). Its height is $1480 \mathrm{~mm}$ with $63.8 \mathrm{~kg}$ weight. So far, WABIAN-2 has realized a human-like walk with the knees stretched, heel-contact and toe-off motion by utilizing a foot mechanism having a passive toe joint and a 2-DOF (Roll, Yaw) waist mimicking a human's pelvic motion. A notable feature of human walking is that the vertical ground reaction force (GRF) has two peaks. Two peaks do not appear when a robot walks with bending the knees. On the other hand, during the knee-stretched walking, we can find two peaks of the vertical ground force which are similar to the human ones as shown in Fig. 2.

WABIAN-2 can contribute to quantitatively evaluate welfare and rehabilitation instruments such as a walk-assist machine, an artificial leg and so on instead 
of human subjects. As one of the applications as the human motion simulator, we carried out walking experiments with a walk-assist machine ${ }^{4}$ (see Fig. 3). Generally, an armrest of a walk-assist machine is set to equal height of a user's elbow. It is also known that the severer his/her disability is, the lower we should set the armrest. Measuring the current value of the actuators at

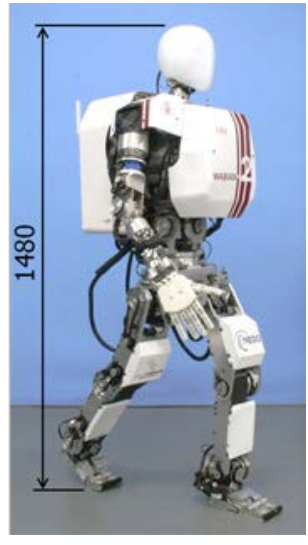

(a) Overview

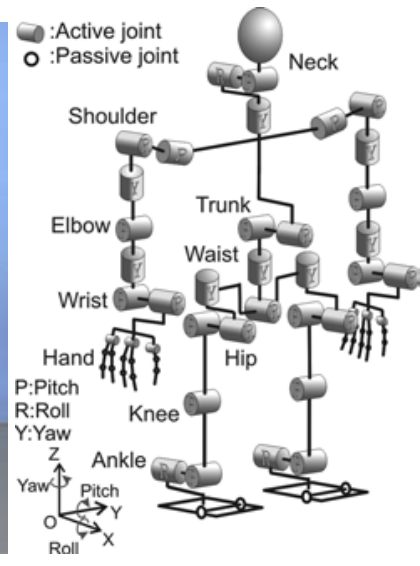

(b) DOF configuration
Fig. 1. Biped humanoid robot, WABIAN-2.

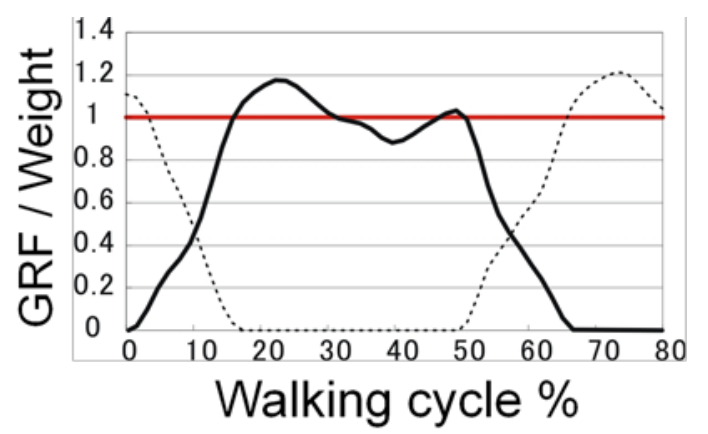

Fig. 2. Ground reaction force during the knee-stretched walking. Two peaks appear like the human ones.

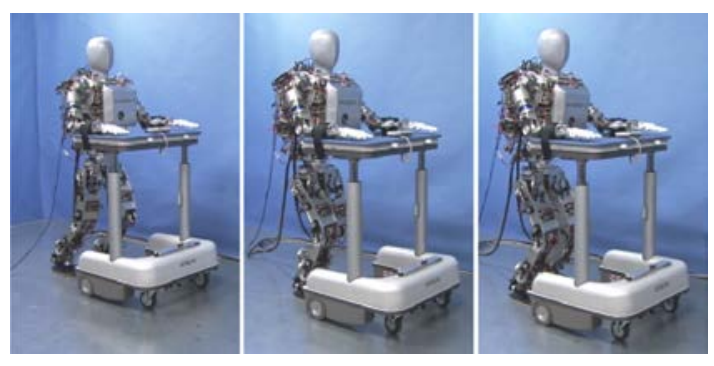

Fig. 3. Quantitative evaluation of walk-assist machine. the knee joints and the forces and torques applied to the arms and legs, we can calculate the energy consumption at knee joints and the load to arms. Then we found the fact with quantitative data that the lower we set the height of the armrest, the less the load of knees is. This is a good example that human motion simulator can evaluate empirical facts quantitatively. From the results of these experiments, we confirmed that we can evaluate welfare and rehabilitation instruments quantitatively with a human motion simulator.

We also use WABIAN-2 to clarify the function of the foot arch structure. ${ }^{5}$ We developed a human-like foot mechanism that mimics the changes of the elasticity of the medial longitudinal arch with the change of the arch height during walking. As experimental results using the biped humanoid robot, WABIAN-2 mounted on the human-like foot mechanism, we confirmed that the arch elasticity could absorb a foot-landing force at the plantar contact phase. Furthermore, the windlass mechanism which caused change of the arch height contributed to a strong thrust at the push-off phase.

\section{Biped Running Robot}

We are now developing a new biped humanoid robot that can mimic various characteristics of human running for investigating human running mechanisms and human motion control. If a robot could perform motions that would improve sports performance, it would be possible to identify more effective human motions and create a novel sports coaching program.

Human running can be modeled by a spring-loaded inverted pendulum (SLIP) model, which is composed of a body mass and spring leg inspired by the linear relationship between the ground reaction force and the vertical body displacement during running. ${ }^{6}$ This model describes human running in a simple, straightforward way and is used on the studies of biped running robots. Moreover, the knee and ankle joints in a standing leg act like torsion springs which emerge the leg stiffness. ${ }^{7}$ Furthermore, an important characteristic that has not yet been reported is that a pelvic movement in the frontal plane can help to increase jumping forces.

On the basis of the analysis of human running motion, we propose a new model named SLIP ${ }^{2}$ (SpringLoaded Inverted Pendulum using Pelvis). This model is 
composed of a body mass, a pelvis, and leg springs. In this model, the knee and ankle joints in a standing leg act like torsion springs and the pelvic sine movement causes the resonance for hopping.

We have developed a biped running robot with a pelvis and a joint stiffness adjustment mechanism that uses two leaf springs mimicking the joint stiffness of a human leg during running (see Fig. 4). The robot has realized hopping motions by utilizing the pelvic movement and leg elasticity.

\section{Biped Vehicle, WL-16}

We consider that a biped vehicle is a viable solution in barrier-free engineering that is much more effective and

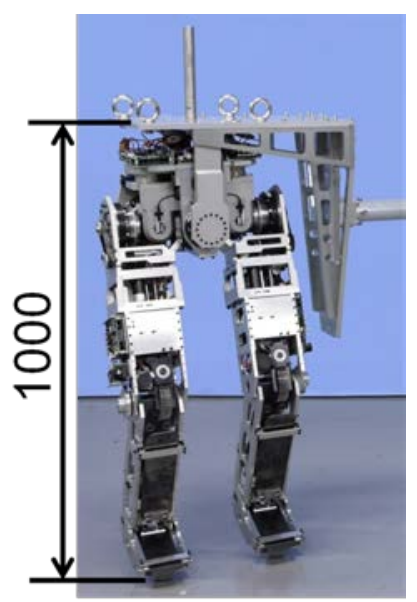

(a) Overview

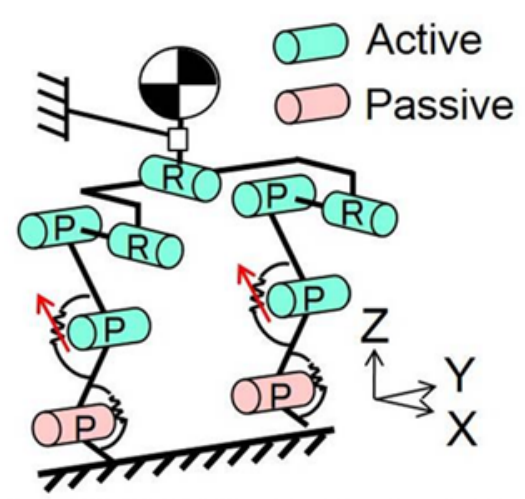

(b) DOF configuration

Fig. 4. Biped running robot. low-cost than infrastructure improvements. Therefore, we have developed a human-carrying biped vehicle named WL-16 as shown in Fig. 5.

The weight of WL-16 is $74 \mathrm{~kg}$ including $11 \mathrm{~kg}$ battery weight. Each leg mechanism has six linear actuators and passive joints at the both sides of each linear actuator. The upper passive joints are commercial universal joints which use needle bearings with little backlash. The lower passive joints are lightweight 3DOF spherical rolling joints, which were newly developed in cooperation with HEPHIST Seiko Co., Ltd.

Each linear actuator consists of a 150W DC servo motor and a ball screw. The control computer is

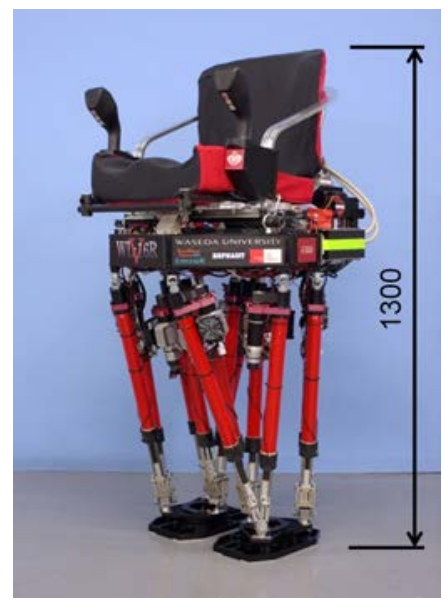

(a) Overview

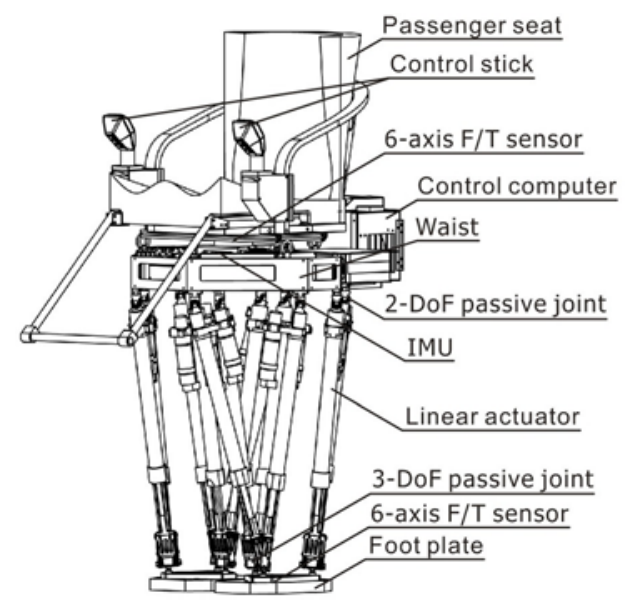

(b) Mechanism and sensors

Fig. 5. Biped vehicle, WL-16. 
arranged at the rear of the waist. The batteries, DC servo drives and an IMU sensor are arranged inside the waist part. A passenger seat is on the waist, and a control stick is mounted on the passenger seat. A passenger can change the direction of the robot freely by using the control stick.

\section{Conclusions}

We introduced our latest biped robots developed at Waseda University. WABIAN-2 is the biped humanoid robot which can walk with the knees stretched, heelcontact and toe-off motion by utilizing a foot mechanism having a passive toe joint and a waist mimicking a human's pelvic motion. WABIAN-2 can quantitatively evaluate welfare and rehabilitation instruments by analyzing the data from a lot of sensors throughout the body. WABIAN-2 also can evaluate ride quality of WL-16 (see Fig. 6). WL-16 is the biped vehicle consisting of two Stewart Platform type legs and waist with a passenger seat. It can be used as a substitute for a wheel chair.

The new biped running robot is designed based on the analysis of human running motion, and it can jump by utilizing the pelvic movement and leg elasticity. We

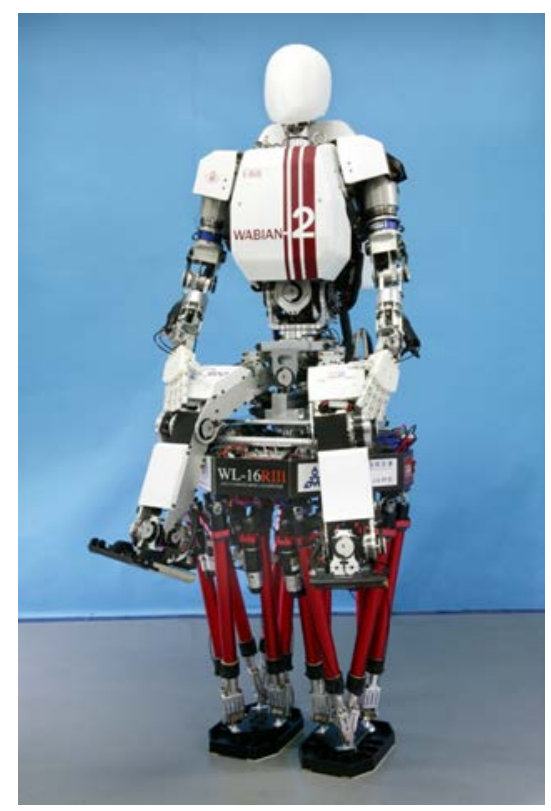

Fig. 6. Cooperative relation between WABIAN-2 and WL-16. will identify more effective human motions by using the running robot and try to create a novel sports coaching program.

\section{Acknowledgements}

This study was conducted as part of the Research Institute for Science and Engineering, Waseda University, and as part of the humanoid project at the Humanoid Robotics Institute, Waseda University. It was also supported in part by MEXT/JSPS KAKENHI (Grant Number: 24360099, 25220005 and 25709019), SolidWorks Japan K.K., HEPHIST Seiko Co., Ltd., and DYDEN Corporation whom we thank for their financial and technical support.

\section{References}

1. Y. Ogura, H. Aikawa, K. Shimomura, H. Kondo, A. Morishima, H. O. Lim and A. Takanishi, Development of a Humanoid Robot WABIAN-2, in Proc. of the 2006 IEEE International Conference on Robotics and Automation (2006) 76-81.

2. T. Otani, M. Yahara, K. Uryu, A. Iizuka, K. Hashimoto, T. Kishi, N. Endo, M. Sakaguchi, Y. Kawakami, S. H. Hyon, H. O. Lim and A. Takanishi, Running Model and Hopping Robot Using Pelvic Movement and Leg Elasticity, in Proc. of the 2014 IEEE International Conference on Robotics and Automation (2014) 23132318.

3. K. Hashimoto, H. O. Lim and A. Takanishi, Disturbance Compensation Control for Biped Vehicle, Advanced Robotics 25 (3) (2011) 407-426.

4. Y. Ogura, H. Aikawa, K. Shimomura, H. Kondo, A. Morishima, H. O. Lim and A. Takanishi, Development of a humanoid robot capable of leaning on a walk-assist machine, in Proc. of the IEEE RAS/EMBS International Conference on Biomedical Robotics and Biomechatronics (2006) 835-840.

5. K. Hashimoto, Y. Takezaki, K. Hattori, H. Kondo, T. Takashima, H. O. Lim and A. Takanishi, A Study of Function of the Human's Foot Arch Structure Using Biped Humanoid Robot, in Proc. of the 2010 IEEE/RSJ International Conference on Intelligent Robots and Systems (2010) pp. 2206-2211.

6. T. McMahon and G. Cheng, The Mechanics of Running: How does Stiffness Couple with Speed?, J. Biomechanics 23 (1990) 65-78.

7. M. Gunther, R. Blickhan, Joint stiffness of the ankle and the knee in running, J. Biomechanics 35 (2002) 1459 1474. 